\title{
Sensoriamento remoto multiespectral na identificação e mapeamento das variáveis bióticas e abióticas do cafeeiro ${ }^{1}$
}

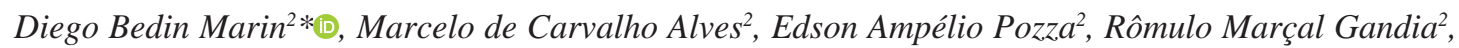 \\ Matheus Luiz Jorge Cortez ${ }^{2}$, Matheus Campos Mattioli ${ }^{2}$
}

10.1590/0034-737X201966020009

\begin{abstract}
RESUMO
O sensoriamento remoto multiespectral apresenta-se como metodologia confiável e viável para auxiliar o produtor na decisão para melhores práticas de manejo, garantindo uma produção agrícola mais eficiente e sustentável. Objetivouse, com este trabalho, identificar e mapear o estresse em lavoura cafeeira, causado por variáveis bióticas e abióticas, por meio de índices de vegetação derivados de imagens multiespectrais Landsat-5 Thematic Mapper (TM). A malha amostral foi composta por 67 pontos, sendo cada ponto amostral constituído por cinco plantas. As análises de incidência de cercosporiose e de infestação do bicho-mineiro, nas folhas, de pH, matéria orgânica e textura do solo e teores foliares de nutrientes foram realizadas em cada um dos pontos amostrais e correlacionadas com 16 índices de vegetação obtidos de imagens referentes à época das análises. Os índices de vegetação apresentaram distribuição espacial semelhante à distribuição espacial das variáveis agronômicas, na lavoura. Houve correlação positiva dos índices com a infestação do bicho-mineiro e com os teores de silte e argila no solo e concentrações de $\mathrm{Mg}, \mathrm{Cu}, \mathrm{B}$ e $\mathrm{Mn}$ nas folhas, e negativa, com a incidência de cercosporiose e com pH e teor de areia do solo. Com base nesses resultados, foi possível mapear e identificar as alterações na reflectância espectral dos cafeeiros, causadas por essas variáveis agronômicas.
\end{abstract}

Palavras-chave: Lansat-5 TM; agricultura de precisão; variáveis agronômicas; índices de vegetação; Coffea arabica L.

\section{ABSTRACT \\ Multispectral remote sensing in the identification and mapping of biotic and abiotic coffee tree variables}

Multispectral remote sensing is a reliable and feasible methodology to assist farmers in decision making for best management practices, ensuring a more efficient and sustainable agricultural production. The objective of this study was to identify and map stress on coffee caused by biotic and abiotic variables through vegetation indices derived from Landsat-5 Thematic Mapper (TM) multispectral images. The sampling grid was composed of 67 points, with each sampling point consisting of five plants. The analyzes of the incidence of brown eye spot and infestation of the leaf miner in the leaves, $\mathrm{pH}$, organic matter, soil texture and nutrients leaf contents were performed at each of the sampling points and correlated with 16 vegetation indices obtained from images at the time of analysis. The vegetation indices presented a spatial distribution similar to the agronomic variables in the crop. There was a positive correlation of the indices with infestation of the leaf miner, silt and clay content in the soil and concentration of $\mathrm{Mg}, \mathrm{Cu}, \mathrm{B}$ and $\mathrm{Mn}$ in the leaves, and negative with the incidence of brown eye spot, $\mathrm{pH}$ and soil sand content. Based on these results, it was possible to map and identify the changes in the spectral reflectance of the coffee trees, caused by these agronomic variables.

Keywords: Landsat-5 TM; precision agriculture; agronomic variables; vegetation indices; Coffea arabica L.

\footnotetext{
Submetido em 26/03/2018 e aprovado em 26/11/2018.

${ }^{1}$ Este trabalho é parte da dissertação de mestrado do primeiro autor

2Universidade Federal de Lavras, Departamento de Engenharia Agrícola e de Fitopatologia, Lavras, Minas Gerais, Brasil. db.marin@hotmail.com; marcelocarvalhoalves@gmail.com; edsonpozza@gmail.com; romagandia@gmail.com; cortez.agro@gmail.com; mattioli-cmatheus@hotmail.com

*Autor para correspondência: db.marin@hotmail.com
}

Rev. Ceres, Viçosa, v. 66, n.2, p. 142-153, mar/abr, 2019 


\section{INTRODUÇÃO}

O café brasileiro é uma commodity de grande impacto no comércio global. O Brasil é o maior produtor e exportador de café, com $36 \%$ da produção mundial, equivalente a $1,98 \mathrm{~T} \mathrm{ha}^{-1}$. (Conab, 2018). No entanto, vários fatores podem reduzir essa produtividade e, por isso, a cafeicultura necessita de uma metodologia robusta, confiável e de baixo custo, de monitoramento de doenças, pragas, estresse hídrico, fertilidade do solo, condições nutricionais e de outros fatores de estresses na cultura (Chemura et al., 2017).

Nos últimos anos, as metodologias de sensoriamento remoto têm sido amplamente utilizadas no monitoramento das culturas agrícolas e na tomada de decisão, para melhores práticas de manejo. Na cultura do café, o uso de dados de sensoriamento remoto provou ser muito promissor, face à dificuldade de se obter dados de campo em escala regional, especialmente para os mapeamentos de campo, que necessitam de mão de obra qualificada, vistorias nas plantas, em lavouras cultivadas em grandes áreas, tempo excessivo de atividade e, consequentemente, aumento do custo de produção (Bernardes et al., 2012).

O sensoriamento remoto espectral fornece a possibilidade de avaliação precoce, eficiente, objetiva e não destrutiva, das respostas das plantas a diferentes fatores de estresse do meio ambiente (Li et al., 2010). As plantas reagem aos estresses biótico e abiótico por meio de mudanças biofísicas e bioquímicas, como reduções de biomassa, do teor de clorofila e alterações nas estruturas internas das folhas, que podem ser facilmente detectadas por meio das diferenças de energia refletida nas regiões espectrais do visível e do infravermelho próximo (Barton, 2012; Mahajan et al., 2014). Além disso, essa diferença de reflectância entre as regiões espectrais do visível e do infravermelho próximo, nas plantas sob estresse, levaram ao desenvolvimento de índices de vegetação (Mirik et al., 2011; Hillnhü̈ter et al., 2011).

Os índices de vegetação são calculados como razões ou diferenças da reflectância de dois ou mais comprimentos de ondas, nas regiões espectrais do visível, dos infravermelhos próximo e médio (Wójtowicz et al., 2016). Esses índices apresentam alta correlação com mudanças na fisiologia e na química das plantas e baixa sensibilidade a fatores responsáveis por dificultar a interpretação de dados obtidos por sensoriamento remoto, por exemplo, sombreamento, cobertura do dossel ou copa da planta, condição e geometria da atmosfera, ângulo solar e fundo do solo (Govender et al., 2009; Mirik et al., 2011; Ortiz et al., 2011).

Em alguns trabalhos, os índices de vegetação mostraram-se confiáveis para avaliar as condições de estresse das lavouras cafeeiras. Por exemplo, Chemura et al. (2016) relataram precisão de $82,5 \%$ na discriminação de diferentes níveis de infecção da ferrugem (Hemileia vastatrix), em lavoura de café (Coffea arabica), utilizando índices de vegetação obtidos a partir de medições feitas por espectro radiômetro. Já Santos et al. (2014), com o auxílio do índice de vegetação EVI-2, derivado do sensor MODIS, encontraram correlação de $\left(R^{2}=0,81\right)$ entre a umidade no perfil do solo, até $100 \mathrm{~cm}$ de profundidade, e o vigor do cafeeiro (Coffea arabica). De forma semelhante, Bernardes et al. (2012) evidenciaram as variações provocadas pela bienalidade na produção de café, em lavoura no sul do Estado de Minas Gerais, entre 2002 e 2009, utilizando os índices de vegetação EVI e NDVI, derivados de imagens MODIS. No entanto, esses estudos não avaliaram o potencial dos índices de vegetação obtidos a partir de imagens Landsat-5 Thematic Mapper (TM), para avaliar as condições do cafeeiro. Além disso, os autores desses estudos concluíram que, apesar do potencial dos índices de vegetação na avaliação das condições do cafeeiro, estudos adicionais ainda são necessários para melhorar a acurácia e precisão da metodologia na avaliação das condições do cafeeiro.

O satélite Landsat-5 TM fornece imagens de média resolução espacial com sete bandas espectrais e resolução espacial de $30 \mathrm{~m}$. O tamanho da imagem da área obtida por esse sensor é de aproximadamente $185 \mathrm{~km}$ e o tempo de revisita do satélite, para obter uma mesma imagem dessa porção do terreno, é de 16 dias (Prabhakar et al., 2012). O Landssat-5 TM também fornece um vasto acervo de imagens prontamente disponíveis e gratuitas. Essas características oferecem vantagens, em relação aos satélites de alta resolução espacial que, além do alto custo de aquisição e armazenamento, demandam elevado tempo de processamento, dificultando a aquisição contínua de dados. Por essa razão, o Landsat-5 TM pode fornecer informações suficientes e baratas para o monitoramento contínuo do estresse, em lavouras cafeeiras, em grandes áreas (Mirik et al., 2013).

Em face do exposto, objetivou-se, por meio de índices de vegetação derivados de imagens multiespectrais Landsat-5 TM, identificar e mapear o estresse em lavoura cafeeira (Coffea arabica L.), causado por variáveis bióticas e abióticas.

\section{MATERIAL E MÉTODOS}

\section{Área de estudo}

O estudo foi realizado na fazenda Cafua, no município de Ijaci, sul de Minas Gerais, em área de 6,5 ha de lavoura de cafeeiro (Coffea arabica L.) do cultivar Mundo Novo, com dez anos, no espaçamento de $4 \mathrm{~m}$ entre 
linhas e $1 \mathrm{~m}$ entre plantas, totalizando 2.500 plantas ha ${ }^{1}$. As coordenadas geográficas são de $21^{\circ} 10^{\prime} 11^{\prime \prime} \mathrm{S}$ e 440 58' 37" O, com altitude média de 934 m e declividade média de $0,84 \%$, no sentido norte-sul, e média de $12 \%$, no sentido leste-oeste. A malha amostral foi composta por 67 pontos georreferenciados, com distâncias de $25 \times 25 \mathrm{~m}$ e $50 \times 50 \mathrm{~m}$ entre si. A opção por uma malha irregular (Alves et al., 2011) ocorreu tanto pela declividade do terreno e do respectivo desenho das curvas de nível quanto pelo número de ruas e carreadores e pela disposição das linhas e do limite da lavoura cafeeira. Cada ponto amostral foi constituído por cinco plantas, quatro plantas distribuídas em torno de uma planta central. O georreferenciamento dos pontos foi realizado com GPS TRIMBLE 4600 LS $®$ e Estação Total Leica TC600®, com base em correção de cotas por coordenadas conhecidas no câmpus da Universidade Federal de Lavras.

\section{Avaliações das variáveis bióticas e abióticas na lavoura}

Avaliações da incidência da cercosporiose (Cercospora coffeicola (Berkeley \& Cooke) e infestação do bicho-mineiro (Leucoptera coffeella) (GuérinMèneville, 1842) (Lepidoptera:Lyonetiidae), nas folhas (\%), foram realizadas observando-se os sinais da doença em 100 folhas coletadas em cada ponto georreferenciado. Cada ponto amostrado consistiu em cinco plantas. As amostras foram coletadas, aleatoriamente, de cada lado da linha de plantação, correspondendo aos pontos cardeais oeste e leste, no terço médio das plantas, com dez folhas amostradas por planta em cada lado da linha de plantação, a partir do terceiro e do quarto par de folhas, contados a partir do final do ramo plagiotrópico, totalizando 6.700 folhas, de acordo com as recomendações de Boldini (2001).

Os teores dos macronutrientes $\mathrm{K}$ e $\mathrm{Mg}\left(\mathrm{g} \mathrm{kg}^{-1}\right)$ e dos micronutrientes $\mathrm{Cu}, \mathrm{B}, \mathrm{Fe}, \mathrm{Mn}\left(\mathrm{mg} \mathrm{dm}^{-3}\right)$, nas folhas, foram avaliados de acordo com Malavolta et al. (1997). Foram amostradas cinco folhas de cada lado da rua, na direção leste e oeste, em três plantas por ponto, totalizando 1.005 folhas. Logo após a coleta, as amostras foram submetidas à limpeza com água corrente e enxaguadas em água deionizada, para remover contaminações na superfície. Em seguida, foram secadas, a $60^{\circ} \mathrm{C}$, em estufa, por 72 horas, e moídas.

As análises de $\mathrm{pH}$, de matéria orgânica $\left(\mathrm{g} \mathrm{kg}^{-1}\right)$ e de teores de areia, silte e argila $\left(\mathrm{g} \mathrm{kg}^{-1}\right)$ do solo foram realizadas na camada de 0-0,20 m, de acordo Cantarutti et al. (1999), na projeção da copa das plantas de cada ponto amostral.

As avaliações da incidência da cercosporiose, infestação do bicho-mineiro e teores de nutrientes, nas folhas, foram realizadas em 29/05/2006, e as análises de $\mathrm{pH}$, matéria orgânica e teores de areia, silte e argila do solo, em 04/ 02/2006

\section{Índices de vegetação}

Para o cálculo dos índices de vegetação, utilizaram-se imagens multiespectrais do satélite Landsat-5 TM Collection 1 Level-2, obtidas nas datas de 26/01/2006 e $18 / 05 / 2006$. Os critérios para escolha dessas datas foram a disponibilidade no acervo, a ausência de nuvens e, principalmente, a proximidade com as datas das análises das variáveis bióticas e abióticas na lavoura. Estas imagens foram obtidas gratuitamente do United States Geological Survey (USGS), em valores de reflectância nas bandas espectrais do azul (450 a $520 \mathrm{~nm})$, do verde $(520$ a $600 \mathrm{~nm})$, do vermelho (630 a $690 \mathrm{~nm}$ ), do infravermelho próximo (760 a $900 \mathrm{~nm})$, do infravermelho médio 1 (1.550 a 1.750 $\mathrm{nm}$ ) e do infravermelho médio 2 (2.080 a $2.350 \mathrm{~nm}$ ). Foi aplicada a correção atmosférica Second Simulation of Satellite Signal in the Solar Spectrum (6S) para minimizar o efeito da atmosfera na reflectância dessas bandas, não sendo necessário realizar o pré-processamento das imagens.

Os índices de vegetação foram calculados em cada pixel das imagens referentes a cada um dos 67 pontos amostrais, baseando-se na combinação dos valores de reflectância nas bandas espectrais das imagens (Tabela 1). A escolha dos dezesseis índices de vegetação foi baseada na capacidade desses em discriminar diferentes características e condições de estresse da vegetação, a partir de dados de sensoriamento remoto (Eitel et al., 2006).

Apesar de a resolução espacial de $30 \mathrm{~m}$ das imagens Landsat-5 TM representar, aproximadamente, 225 plantas, e de cada análise das variáveis bióticas e abióticas, nos pontos amostrais, cinco plantas, a utilização dessas imagens torna-se possível. Conforme demonstrado por Alves et al. (2011) e Alves (2006), em estudo sobre a distribuição espacial das variáveis utilizadas neste estudo, por meio da krigagem, a dependência espacial dessas é, em média, de $30 \mathrm{~m}$. Portanto, a informação das 225 plantas que o satélite capta em cada pixel pode representar o que foi analisado em somente cinco plantas.

\section{Análise de correlação}

Os dados de análise da incidência da cercosporiose, infestação do bicho-mineiro e teor de nutrientes, nas folhas, na data de 29/05/2006, e os valores dos índices de vegetação, na data de 18/05/2006, calculados para cada pixel referente a cada um dos 67 pontos amostrais, foram submetidos à análise de correlação de Pearson $(\mathrm{p}<0,01)$. Os dados de $\mathrm{pH}$, matéria orgânica e teor de areia, silte e argila do solo, na data de 04/02/2006, foram correla- 
cionados com os valores dos índices de vegetação, na data de 26/01/2006.

\section{RESULTADOS E DISCUSSÕES}

\section{Distribuição espacial das variáveis bióticas e abióticas e dos índices de vegetação}

Os mapas de distribuição espacial da incidência de cercosporiose e da infestação do bicho-mineiro, nas folhas, de 29/05/2006, apresentaram correspondência com os mapas de distribuição espacial dos índices de vegetação em 18/05/2006 (Figuras 1 e 2). Foi possível observar a correspondência inversa da incidência de cercosporiose, e, direta, da infestação do bicho-mineiro, nas folhas, com os índices de vegetação.
A correspondência entre a distribuição espacial de doenças em plantas e os índices de vegetação também foi relatada por Martins \& Galo (2014). Segundo esses autores, a distribuição espacial de nematoides e do besouro Migdollus fryanus, em lavoura de cana-de-açúcar, apresentaram correspondência direta com as distribuições espaciais de NDVI, EVI e SAVI. De forma semelhante, Motomiya et al. (2012) relataram distribuição espacial com correspondência entre o NDVI e o ataque do percevejo castanho [Scaptocoris castanea (Hem.: Cydnidae)] em lavoura de algodão.

Para os mapas de distribuição espacial das características físicas e químicas do solo, em 04/02/2006, houve correspondência direta da distribuição espacial dos índices de vegetação em 26/01/2006, com os teores de matéria

Tabela 1: Índices de vegetação obtidos a partir da reflectância das bandas multiespectrais das imagens Landsat-5 TM

\begin{tabular}{|c|c|c|}
\hline Índices de Vegetação & Equação & Referência \\
\hline \multirow{2}{*}{ OSAVI (Optmized SAVI) } & $(1+0.16)\left(\rho_{\text {nir }}-\rho_{\text {red }}\right)$ & \multirow{2}{*}{ Rondeaux et al. (1996) } \\
\hline & $\rho_{\text {nir }}+\rho_{\text {red }}+0.16$ & \\
\hline SIPI (Structural Insensitive Pigment Index) & $\frac{\rho_{\text {nir }}-\rho_{\text {blue }}}{\rho_{\text {nir }}+\rho_{\text {red }}}$ & Peñuelas et al. (1995) \\
\hline \multirow{2}{*}{$\begin{array}{l}\text { GARI (Green Atmospherically Resistant } \\
\text { Vegetation Index) }\end{array}$} & $\rho_{\text {nir }}-\left[\rho_{\text {oreen }}-1.7\left(\rho_{\text {hlue }}-\rho_{\text {red }}\right]\right)$ & \multirow[b]{2}{*}{ Gitelson et al. (1996) } \\
\hline & {$\left[\rho_{\text {nir }}+\left(\rho_{\text {green }}-1.7\left(\rho_{\text {blue }}-\rho_{\text {red }}\right]\right)\right.$} & \\
\hline TVI (Triangular Vegetation Index) & $0.5\left[120\left(\rho_{\text {nir }}-\rho_{\text {oreen }}\right)-200\left(\rho_{\text {red }}-\rho_{\text {ore }}\right.\right.$ & \\
\hline MCDU1 (Fi' & 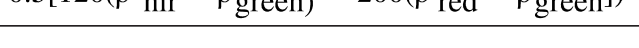 & 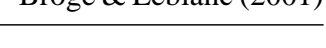 \\
\hline $\begin{array}{l}\text { MCARI1 (First Modified Chlorophyll } \\
\text { Absorption Ratio Index) }\end{array}$ & $1.2\left[2.5\left(\rho_{\text {nir }}-\rho_{\text {green }}\right)-1.3\left(\rho_{\text {nir }}-\rho_{\text {green }}\right)\right]$ & Haboudane et al. (2004) \\
\hline \multirow{2}{*}{$\begin{array}{l}\text { MCARI2 (Second Modified Chlorophyll } \\
\text { Absorption Ratio Index) }\end{array}$} & $1.2\left[2.5\left(\rho_{\text {nir }}-\rho_{\text {red }}\right)-1.3\left(\rho_{\text {nir }}-\rho_{\text {green }}\right)\right]$ & \multirow{2}{*}{ Haboudane et al. (2004) } \\
\hline & $\sqrt{2\left(\rho_{\text {nir }}+1\right)^{2}}-0.5-6\left(\rho_{\text {nir }}-5 \sqrt{\rho_{\text {red }}}\right)$ & \\
\hline MSAVI (Modified SAVI) & $0.5\left[2 \rho_{\text {nir }}+1-\sqrt{\left.\left(2 \rho_{\text {nir }}+1\right)^{2}-8\left(\rho_{\text {nir }}-\rho_{\text {red }}\right]\right)}\right.$ & Qi et al. (1994) \\
\hline NDWI (Normalized Difference Water Index) & $\frac{\rho_{\text {nir }}-\rho_{\text {mid }}}{\rho_{\text {nir }}+\rho_{\text {mid }}}$ & Gao (1996) \\
\hline NDVI (Normalized Difference Vegetation Index) & $\frac{\rho_{\text {nir }}-\rho_{\text {red }}}{\rho_{\text {nir }}+\rho_{\text {red }}}$ & Rouse et al. (1974) \\
\hline \multirow{2}{*}{ SAVI (Soil Adjusted Difference Vegetation Index) } & $(1+L) \rho_{\text {nir }}-\rho_{\text {red }}$ & \multirow[b]{2}{*}{ Huete (1988) } \\
\hline & $\rho_{\text {nir }}+\rho_{\text {red }}+L$ & \\
\hline \multirow{2}{*}{$\begin{array}{l}\text { GNDVI (Green Normalized Difference } \\
\text { Vegetation Index }\end{array}$} & $\rho_{\text {nir }}-\rho_{\text {green }}$ & \multirow{2}{*}{ Gitelson et al. (1996) } \\
\hline & $\overline{\rho_{\text {nir }}+\rho_{\text {green }}}$ & \\
\hline $\begin{array}{l}\text { MTVI }_{1} \text { (Modified Normalized Difference } \\
\text { Vegetation Index 1) }\end{array}$ & $1.2\left[1.2\left(\rho_{\text {nir }}-\rho_{\text {green }}\right)-2.5\left(\rho_{\text {red }}-\rho_{\text {green }}\right]\right)$ & Haboudane et al. (2004) \\
\hline \multirow{2}{*}{$\begin{array}{l}\text { MTVI }_{2} \text { (Modified Normalized Difference } \\
\text { Vegetation Index 2) }\end{array}$} & $1.5\left[1\left(\rho_{\text {nir }}-\rho_{\text {green }}\right)-2.5\left(\rho_{\text {red }}-\rho\right.\right.$ green $\left.)\right]$ & \multirow{2}{*}{ Haboudane et al. (2004) } \\
\hline & $\sqrt{\left[\left(2 \rho_{\text {nir }}+1\right)^{2}-\left(6 \rho_{\text {nir }}-5 \sqrt{\rho_{\text {red }}}-0.5\right)\right]}$ & \\
\hline RDVI (Renormalized Difference Vegetation Index) & $\sqrt{\frac{\rho_{\text {nir }}-\rho_{\text {red }}}{\rho_{\text {nir }}+\rho_{\text {red }}}}$ & Roujean \& Breon (1995) \\
\hline \multirow{2}{*}{ MSR (Modified SR) } & $\left(\frac{\rho_{\text {nir }}}{\rho_{\text {red }}}\right)-1$ & \multirow{2}{*}{ Chen (1996) } \\
\hline & $\sqrt{\left(\frac{\rho_{\text {nir }}}{\left.\rho_{\text {red }}\right)}+1\right.}$ & \\
\hline SR (Simple Ratio) & $\frac{\rho_{\text {nir }}}{\rho_{\text {red }}}$ & Birth \& McVey (1968) \\
\hline
\end{tabular}



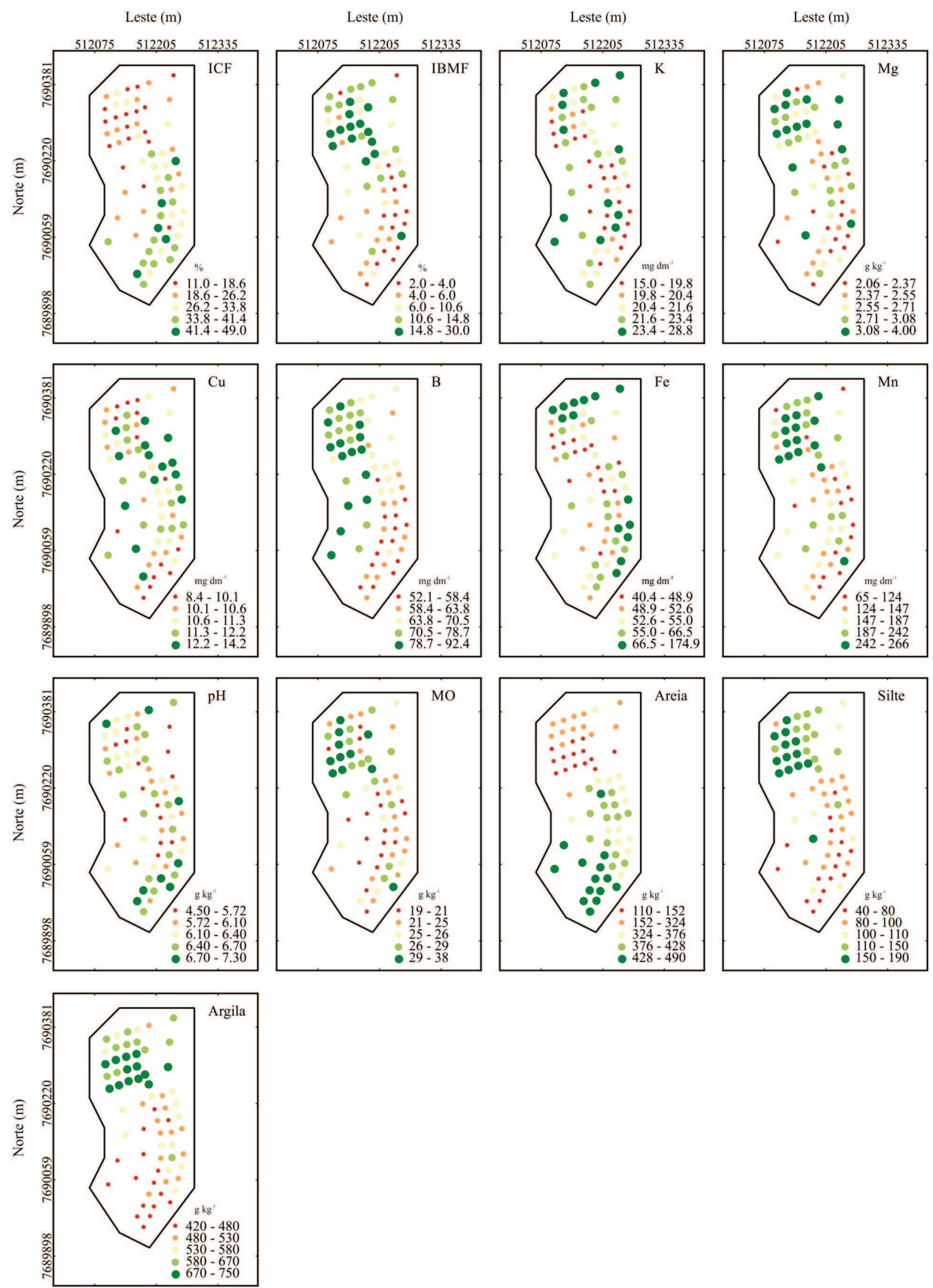

Figura 1: Mapas de quintil da distribuição espacial, em lavoura cafeeira (Coffea arabica), da incidência da cercosporiose nas folhas (ICF), infestação do bicho-mineiro, potássio (K), magnésio (Mg), cobre (Cu), boro (B), ferro (Fe) e manganês (Mn), em 29/05/2006, e do pH em água, matéria orgânica (Mo), areia, silte e argila, em 04/02/2006. 

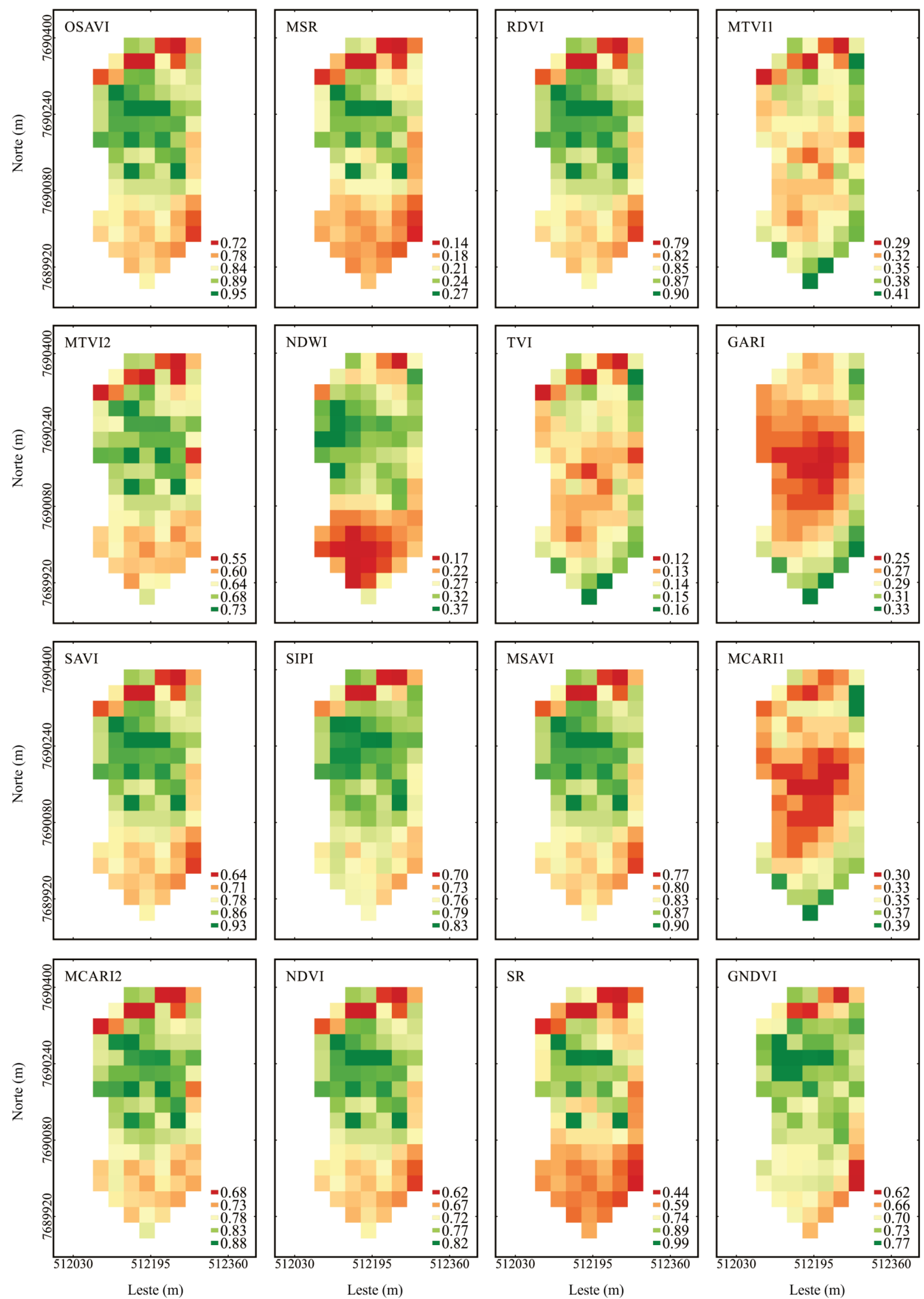

Figura 2: Mapas de distribuição espacial dos índices de vegetação, em lavoura cafeeira (Coffea arabica), derivados de imagem multiespectral Landsat-5 TM, na data de 18/05/2006. 

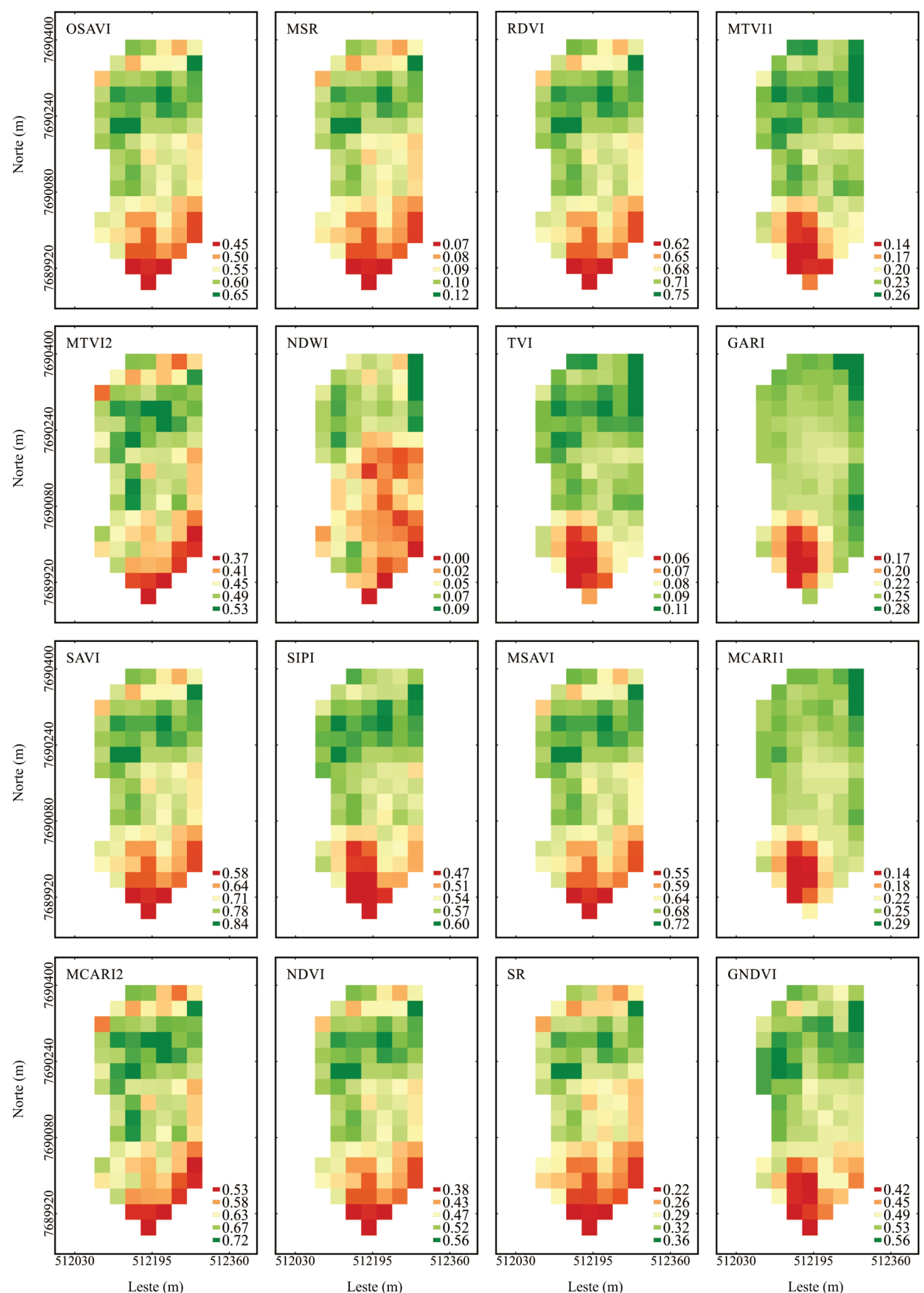

Figura 3: Mapas de distribuição espacial dos índices de vegetação, em lavoura cafeeira (Coffea arabica), derivados de imagem multiespectral Landsat-5 TM, na data de 26/01/2006.

Rev. Ceres, Viçosa, v. 66, n.2, p. 142-153, mar/abr, 2019 
orgânica, silte e argila, e correspondência inversa com pH e o teor de areia (Figuras 1 e 3).

Estudando a distribuição espacial das características do solo, em um sistema integrado de lavoura e pecuária, Bernardi et al. (2017) encontraram correspondência entre o NDVI e os parâmetros do solo pH e teores de matéria orgânica, areia e argila. Já Zanzarini et al. (2013) relataram correspondência do NDVI com a distribuição de argila em um Latossolo Vermelho-Amarelo em área com cana-deaçúcar. No entanto, ressalte-se que esses estudos não exploraram o potencial de outros índices de vegetação na relação com as características do solo, como é o caso deste estudo.

Com relação aos mapas de distribuição espacial dos macro e micronutrientes, nas folhas, em 29/05/2006, houve correspondência direta da distribuição espacial dos índices de vegetação, em 18/05/2006, com os teores de $\mathrm{Mg}, \mathrm{Cu}, \mathrm{B}$ e $\mathrm{Mn}$, e correspondência indireta com $\mathrm{K}$ e Fe (Figuras 1 e 2).

\section{Análise de correlações entre as variáveis bióticas e abióticas e os índices de vegetação}

A variável incidência da cercosporiose nas folhas apresentou coeficientes de correlação negativos com os índices de vegetação e, em contrapartida, coeficientes positivos com a variável infestação do bicho-mineiro, nas folhas (Tabela 2).

De maneira geral, os valores dos coeficientes de correlação entre os índices de vegetação e a incidência da

Tabela 2: Coeficientes de correlação de Pearson $(\mathrm{p}<0,01)$, entre as variáveis incidência da cercosporiose nas folhas e incidência do bicho-mineiro, nas folhas em lavoura de cafeeiro (Coffea arabica), em 29/05/2006, e índices de vegetação, em 18/05/2006

\begin{tabular}{lcc}
\hline $\begin{array}{l}\text { Índices } \\
\text { de vegetação }\end{array}$ & $\begin{array}{c}\text { Incidência da } \\
\text { cercosporiose } \\
\text { nas folhas }\end{array}$ & $\begin{array}{c}\text { Infestação do } \\
\text { bicho-mineiro } \\
\text { nas folhas }\end{array}$ \\
\hline OSAVI & $-0,51$ & 0,40 \\
MSR & $-0,51$ & 0,38 \\
RDVI & $-0,51$ & 0,35 \\
MTVI1 & $-0,57$ & 0,34 \\
NDWI & $-0,61$ & 0,40 \\
MTVI2 & $-0,47$ & 0,31 \\
TVI & $-0,57$ & 0,32 \\
GARI & $-0,47$ & 0,35 \\
SAVI & $-0,51$ & 0,36 \\
SIPI & $-0,59$ & 0,32 \\
MSAVI & $-0,51$ & 0,35 \\
MCARI1 & $-0,53$ & 0,33 \\
MCARI2 & $-0,47$ & 0,32 \\
NDVI & $-0,51$ & 0,36 \\
SR & $-0,51$ & 0,38 \\
GNDVI & $-0,51$ & 0,42 \\
\hline
\end{tabular}

cercosporiose e a infestação do bicho-mineiro, nas folhas, foram semelhantes em cada variável independente. $O$ índice NDWI apresentou o maior valor de coeficiente de correlação $(r=-0,61)$ com a incidência da cercosporiose, enquanto o GNDVI o maior $(r=0,42)$, para a infestação do bicho-mineiro.

As correlações entre os índices de vegetação e a incidência da cercosporiose e a infestação do bicho-mineiro, nas folhas, podem estar associadas com o vigor vegetativo das plantas com a presença desses organismos-praga. Segundo Ahamed et al. (2011), quanto maior o vigor vegetativo das plantas e, consequentemente, maior a quantidade de folhas, menor a reflectância nas bandas do visível (azul, verde e vermelho) e maior na banda do infravermelho próximo. Além disso, a quantidade de folhas aumenta o teor de água na planta, provocando menor refletância na região do infravermelho médio (Moreira et al., 2004). Por isso, a correlação negativa entre os índices de vegetação e a incidência da cercosporiose, nas folhas, pode ser explicada em razão do menor vigor vegetativo das plantas com a presença dessa doença. De acordo com Pozza et al. (2010), a cercosporiose provoca a produção em excesso de etileno, nas folhas lesionadas, sendo este o percursor de outro hormônio, o ácido abscísico, o qual provoca a queda precoce das folhas, diminuindo consideravelmente o vigor vegetativo. Portanto, os valores dos índices de vegetação tendem a diminuir, em plantas com presença de cercosporiose, pois esses são calculados a partir das diferenças de reflectância entre a banda do infravermelho próximo e as bandas do visível e do infravermelho médio, diferentemente dos valores dos índices de vegetação, em plantas infestadas por bicho-mineiro que aumentam com o número de mariposas dessa praga, o que as leva a selecionar plantas com maior vigor vegetativo para a ovoposição (Nestel et al., 1994).

A correlação negativa entre a incidência da cercosporiose e os índices de vegetação também pode estar associada com teores de água nas plantas, pois alguns autores (Santos et al., 2004; Paiva et al., 2013; Vasco et al., 2015) destacam que os maiores índices de cercosporiose são encontrados em parcelas não irrigadas, indicando influência positiva da disponibilidade de água para a planta, na redução da incidência da doença. Por essa razão, os maiores valores de coeficientes de correlação apresentados por NDWI $(r=-0,61)$ podem estar relacionados com a capacidade desse índice para detectar estresse hídrico nas plantas, uma vez que, para o cálculo do NDWI, utiliza-se a banda do infravermelho médio que é sensível aos teores de água nas plantas.

Os valores de coeficientes de correlação entre as características físicas, químicas e biológicas e os índices de vegetação foram positivos para matéria orgânica, silte e argila e negativos para $\mathrm{pH}$ e areia (Tabela 3 ). 
Assim como para as variáveis incidência da cercosporiose e infestação do bicho-mineiro, os valores de coeficiente de correlação entre os índices de vegetação e as características do solo foram semelhantes para as variáveis matéria orgânica, areia, silte e argila, de forma independente. $\mathrm{O}$ índice NDWI apresentou o maior valor de coeficiente de correlação com matéria orgânica $(r=0,58)$, areia $(\mathrm{r}=-0,80)$, silte $(\mathrm{r}=0,75)$ e argila $(\mathrm{r}=0,74)$, e os índices MTVI2 $(r=0,39)$ e MCARI $2(r=0,39)$, com a variável $\mathrm{pH}$ do solo.

A correlações positivas entre os índices de vegetação com os teores de matéria orgânica, silte e argila no solo, e negativa, com os teores de areia no solo podem estar associadas com a disponibilidade de nutrientes para as plantas. A textura do solo (areia, silte e argila) é um dos principais fatores responsáveis pela disponibilidade de nutrientes para as plantas. Solos com maiores teores de areia tendem a lixiviar nutrientes com mais facilidade, como no caso do K (Melo et al., 2004), por causa do tamanho de seus poros. Em contrapartida, as frações silte e argila podem contribuir para aumentar as concentrações de $\mathrm{Ca}, \mathrm{Mg}$ e $\mathrm{K}$ na solução do solo (Corrêa et al. 2003). Além disso, a capacidade de troca de cátions (CTC) da matéria orgânica é fundamental para a CTC total do solo, sendo essencial na retenção de nutrientes e na diminuição da lixiviação (Silva et al., 2013). Assim, de maneira geral, solos siltosos, argilosos e com teores de matéria orgânica elevados, propiciam melhores condições para o desenvolvimento das plantas que os solos arenosos. Por isso, nos lugares das lavouras com maiores teores de matéria orgânica, silte e argila no solo, os cafeeiros, provavelmente, apresentavam maior vigor vegetativo e, consequentemente, maiores valores dos índices de vegetação. Em contrapartida, nos lugares com maiores teores de areia, os cafeeiros apresentavam menor vigor vegetativo e menores valores dos índices de vegetação.

Ainda sobre a textura do solo, a sensibilidade do NDWI à presença de água nas plantas pode ter contribuído para os valores do coeficiente de correlação desse índice com a textura do solo maiores do que os dos outros índices, pois, conforme observado no estudo de Arruda et al. (1987), os teores de silte e argila são relacionados com a retenção de água no solo e, portanto, com a maior disponibilidade de água para as plantas.

No caso do pH do solo, a correlação negativa com os índices de vegetação pode ter ocorrido em razão da predominância de valores de $\mathrm{pH}$, na lavoura, acima de 6,5 (Figura 1), visto que a disponibilidade de nutrientes para as plantas em solo com $\mathrm{pH}$ nessa faixa é menor (Moreira \& Fageria, 2010). Dessa forma, regiões da lavoura com $\mathrm{pH}$ acima de 6,5 podem ter contribuído para o menor vigor vegetativo dos cafeeiros e, consequentemente, menores valores dos índices de vegetação.

Os valores dos coeficientes de correlação entre os índices de vegetação, o pH e a textura do solo, obtidos neste estudo, mostraram-se superiores aos encontrados por Tola et al. (2017). No estudo da produtividade da grama Rhodes na região oriental da Arábia Saudita, esses autores evidenciaram correlações negativas do NDVI com o $\mathrm{pH}(\mathrm{r}=-0,11)$ e positiva com silte $(\mathrm{r}=0,32)$, argila $(\mathrm{r}=$ $0,24)$ e areia $(r=0,17)$. Contudo, ressalte-se que a baixa variabilidade espacial de areia na área de estudo desses autores pode ter contribuído para a correlação positiva com o NDVI.

Tabela 3: Coeficientes de correlação de Pearson $(\mathrm{p}<0,01)$ entre as características físicas, químicas e biológicas do solo, em lavoura de cafeeiro (Coffea arabica), em 04/02/2006, e índices de vegetação, em 26/01/2006

\begin{tabular}{|c|c|c|c|c|c|}
\hline Índices de Vegetação & pH & Matéria Orgânica & Areia & Silte & Argila \\
\hline OSAVI & $-0,34$ & 0,43 & $-0,71$ & 0,64 & 0,65 \\
\hline MSR & $-0,34$ & 0,44 & $-0,72$ & 0,65 & 0,66 \\
\hline RDVI & $-0,35$ & 0,43 & $-0,71$ & 0,64 & 0,65 \\
\hline MTVI1 & $-0,31$ & 0,41 & $-0,71$ & 0,64 & 0,66 \\
\hline NDWI & $-0,13$ & 0,58 & $-0,80$ & 0,75 & 0,74 \\
\hline MTVI2 & $-0,39$ & 0,30 & $-0,58$ & 0,54 & 0,53 \\
\hline TVI & $-0,29$ & 0,42 & $-0,72$ & 0,63 & 0,66 \\
\hline GARI & $-0,15$ & 0,34 & $-0,56$ & 0,47 & 0,53 \\
\hline SAVI & $-0,34$ & 0,43 & $-0,71$ & 0,64 & 0,65 \\
\hline SIPI & $-0,29$ & 0,52 & $-0,79$ & 0,69 & 0,73 \\
\hline MSAVI & $-0,35$ & 0,42 & $-0,70$ & 0,64 & 0,64 \\
\hline MCARI1 & $-0,20$ & 0,44 & $-0,69$ & 0,59 & 0,64 \\
\hline MCARI2 & $-0,39$ & 0,33 & $-0,61$ & 0,57 & 0,56 \\
\hline NDVI & $-0,34$ & 0,43 & $-0,71$ & 0,64 & 0,65 \\
\hline SR & $-0,33$ & 0,45 & $-0,73$ & 0,66 & 0,67 \\
\hline GNDVI & $-0,24$ & 0,52 & $-0,75$ & 0,67 & 0,69 \\
\hline
\end{tabular}

Rev. Ceres, Viçosa, v. 66, n.2, p. 142-153, mar/abr, 2019 
Tabela 4: Coeficientes de correlação de Pearson ( $\mathrm{p}<0,01)$, entre macros e micronutrientes, nas folhas, em lavoura de cafeeiro (Coffea arabica), em 29/05/2006, e índices de vegetação, em 18/05/2006

\begin{tabular}{lcccccc}
\hline Índices de vegetação & $\mathbf{K}$ & $\mathbf{M g}$ & $\mathbf{C u}$ & $\mathbf{B}$ & $\mathbf{F e}$ & $\mathbf{M n}$ \\
\hline OSAVI & $-0,306$ & 0,407 & 0,561 & 0,326 & $-0,748$ & 0,624 \\
MSR & $-0,270$ & 0,431 & 0,591 & 0,379 & $-0,710$ & 0,627 \\
RDVI & $-0,312$ & 0,402 & 0,552 & 0,317 & $-0,752$ & 0,623 \\
MTVI1 & $-0,403$ & 0,017 & 0,182 & 0,234 & $-0,650$ & 0,568 \\
NDWI & $-0,146$ & 0,511 & 0,403 & 0,426 & $-0,666$ & 0,561 \\
MTVI2 & $-0,356$ & 0,353 & 0,545 & 0,292 & $-0,732$ & 0,602 \\
TVI & $-0,381$ & 0,012 & 0,115 & 0,279 & $-0,637$ & 0,544 \\
GARI & 0,006 & 0,299 & 0,468 & 0,508 & $-0,261$ & 0,094 \\
SAVI & $-0,303$ & 0,407 & 0,561 & 0,326 & $-0,748$ & 0,624 \\
SIPI & $-0,289$ & 0,389 & 0,513 & 0,301 & 0,740 & 0,559 \\
MSAVI & $-0,316$ & 0,398 & 0,546 & 0,311 & $-0,753$ & 0,623 \\
MCARI1 & $-0,135$ & 0,164 & 0,286 & 0,468 & $-0,528$ & 0,110 \\
MCARI2 & $-0,352$ & 0,365 & 0,535 & 0,298 & $-0,739$ & 0,611 \\
NDVI & $-0,303$ & 0,407 & 0,561 & 0,326 & $-0,748$ & 0,624 \\
SR & $-0,251$ & 0,436 & 0,595 & 0,402 & $-0,679$ & 0,629 \\
GNDVI & $-0,185$ & 0,381 & 0,435 & 0,270 & $-0,709$ & 0,322 \\
\hline
\end{tabular}

Os valores de coeficientes de correlação entre os nutrientes nas folhas e os índices de vegetação foram positivos para $\mathrm{Mg}, \mathrm{Cu}, \mathrm{B}$ e Mn e negativos para K e Fe (Tabela 4).

Apesar de a maioria dos índices de vegetação terem apresentado valores de coeficientes de correlação semelhantes com as características do solo, o índice MTVI1 apresentou o maior valor de coeficiente para $\mathrm{K}(\mathrm{r}=-0,403)$, o NDWI, para Mg $(\mathrm{r}=0,511)$, o SR, para Cu $(\mathrm{r}=0,595) \mathrm{e}$ $\mathrm{Mn}(\mathrm{r}=0,629)$, o GARI, para B $(\mathrm{r}=0,508)$ e o MSAVI, para $\mathrm{Fe}(\mathrm{r}=-0,753)$.

As correlações negativas dos índices de vegetação com os nutrientes $\mathrm{K}$ e Fe nas folhas podem estar associadas ao estresse dos cafeeiros provocado por desequilíbrio nutricional desses nutrientes. As plantas em condições de estresse reduzem a concentração de clorofila nas folhas, apresentam clorose e, consequentemente, diminuem a absorção de luz incidente na região espectral do visível (Carter \& Knapp 2001; Zhao et al., 2003; Bratasevec et al., 2013). Portanto, os cafeeiros com desequilíbrios desses nutrientes podem ter apresentado maiores reflectâncias nas bandas do visível e, dessa forma, reduzido os valores dos índices de vegetação. No caso da correlação positiva dos índices de vegetação, com os nutrientes $\mathrm{Mg}, \mathrm{Cu}, \mathrm{B}$ e $\mathrm{Mn}$, o equilíbrio nutricional desses nutrientes nos cafeeiros pode ter provocado o aumento da concentração de clorofila nas folhas e a redução da reflectância nas bandas do visível, aumentando assim os valores dos índices de vegetação.

Pimstein et al. (2011), monitorando o estresse na cultura do trigo, também observaram correlações negativas de até $\mathrm{r}=-0,45$ dos nutrientes $\mathrm{N}, \mathrm{P}$ e K com a reflectância das plantas nos comprimentos de onda do visível. Esses autores concluíram que monitoramento de nutrientes em cultura pode ser usado com auxílio de dados de sensoriamento remoto. De forma semelhante, Mahajan et al. (2016) demonstraram que os índices de vegetação podem ser usados na predição e monitoramento das condições de N, P e S, na cultura do arroz. Para isso, os autores reportaram correlações do N, Pe S com os índices NDVI ( $\mathrm{r}$ $=0,58,0,34$ e 0,37, respectivamente) e GNDVI $(r=0,47$, 0,29 e 0,33, respectivamente). Entretanto, Serrano et al. (2017) obtiveram correlações não significativas entre o NDVI e os teores de $\mathrm{Na}$ e $\mathrm{K}$, em vinhedo. Os autores explicaram que esse comportamento é aceitável, visto que as plantas analisadas apresentavam concentrações adequadas desses nutrientes, e ainda, ponderam que se espera que, em situações de deficiência de nutrientes de plantas, o vigor vegetativo da planta seja afetado (por exemplo, clorose das folhas), fenômeno esse detectado por sensores remotos.

Por essas razões, foi possível inferir que, as plantas da lavoura de estudo apresentavam deficiência nutricional de $\mathrm{Mg}, \mathrm{Cu}, \mathrm{B}$ e $\mathrm{Mn}$ e equilíbrio de $\mathrm{K}$ e Fe, na época das análises.

\section{CONCLUSÕES}

Este estudo mostrou o potencial dos índices de vegetação derivados de imagens multiespectrais Landsat-5 TM, para identificação e mapeamento do estresse nas plantas de café, causado por variáveis ambientais bióticas e abióticas. Além disso, os resultados deste estudo podem contribuir para um gerenciamento mais eficiente das lavouras cafeeiras, assim como para a sustentabilidade da atividade por meio do uso mais racional de fertilizantes e de produtos fitossanitários. 


\section{REFERÊNCIAS}

Ahamed T, Tian L, Zhang Y \& Ting KC (2011) A review of remote sensing methods for biomass feedstock production. Biomass and Bioenergy, 35:2455-2469.

Alves MC (2006) Geoestatística e sistemas 'fuzzy' na proteção de plantas. Tese de Doutorado. Universidade Federal de Lavras, Lavras. 186p

Alves MC, Silva FM, Moraes JC, Pozza EA, Oliveira MS, Souza JC \& Alves LS (2011) Geostatistical analysis of the spatial variation of the berry borer and leaf miner in a coffee agroecosystem. Precision Agriculture, 12:18-31.

Arruda FB, Zullo Junior J \& Oliveira JB (1987) Parâmetros de solo para o cálculo da água disponível com base na textura do solo. Revista Brasileira de Ciência do Solo, 11:11-15.

Barton CVM (2012) Advances in remote sensing of plant stress. Plant and Soil, 354:41-44.

Bernardes T, Moreira MA, Adami M, Giarolla A \& Rudorff BFT (2012) Monitoring biennial bearing effect on coffee yield using MODIS remote sensing imagery. Remote Sensing, 04:2492-2509.

Bernardi ACC, Célia RG, Ricardo GA, Ladislau MR \& Ricardo YI (2017) Spatial variability of vegetation index and soil properties in an integrated crop-livestock system. Revista Brasileira de Engenharia Agrícola e Ambiental, 21:513-518.

Birth GS \& Mcvey G (1968) Measuring the colour of growing turf with a reflectance spectrophotometer. Agronomy Journal, 60:640-643.

Boldini JM (2001) Epidemiologia da ferrugem e da cercosporiose em cafeeiro irrigado e fertirrigado. Dissertação de Mestrado. Universidade Federal de Lavras, Lavras. 67p.

Bratasevec K, Sivilotti P \& Vodopivec BM (2013) Soil and foliar fertilization affects mineral contents in Vitis vinifera L. cv. 'rebula' leaves. Journal of soil science and plant nutrition, 13:650663.

Broge NH \& Leblanc E (2001) Comparing prediction power and stability of broadband and hyperspectral vegetation indices for estimation of green leaf area index and canopy chlorophyll density. Remote Sensing of Environment, 76:156-172.

Cantarutti RB, Alvarez V \& Ribeiro AC (1999) Amostragem do solo. In: Ribeiro AC, Guimarães PTG \& Alvarez V (Eds.) Recomendação para uso de corretivos e fertilizantes em Minas Gerais: 5 $5^{\text {a }}$ aproximação. Viçosa, CFSEMG. p.13-20.

Carter GA \& Knapp AK (2001) Leaf optical properties in higher plants: linking spectral characteristics to stress and chlorophyll concentration. American journal of botany, 88:677-684.

Conab (2018) Acompanhamento da safra brasileira: grãos. Disponível em: https://www.conab.gov.br/component/k2/ item/download/22249_796d70cec80b021e204b5514764e77b4. Acessado em: 08 de novembro de 2018.

Corrêa MM, Ker JC, Mendonça ES, Ruiz HA \& Bastos RS (2003) Physical, chemical and mineralogical characteristics of soils from the meadow region of sousa (PB). Revista Brasileira de Ciência do Solo, 27:311-324.

Chemura A, Mutanga O \& Dube T (2016) Separability of coffee leaf rust infection levels with machine learning methods at Sentinel-2 MSI spectral resolutions. Precision Agriculture, $18: 859-881$

Chemura A, Mutanga $O$ \& Dube T (2017) Integrating age in the detection and mapping of incongruous patches in coffee (Coffea arabica) plantations using multi-temporal Landsat 8 NDVI anomalies. International Journal of Applied Earth Observation and Geoinformation, 57:01-13.
Chen JM (1996) Evaluation of vegetation indices and a modified simple ratio for boreal applications. Canadian Journal of Remote Sensing, 22:229-242.

Eitel JU, Gessler PE, Smith AM \& Robberecht R (2006) Suitability of existing and novel spectral indices to remotely detect water stress in Populus spp. Forest Ecology and Management, 229:170-182.

Gao B (1996) NDWI - A normalized difference water index for remote sensing of vegetation liquid water from space. Remote sensing of environment, 58:257-266.

Gitelson AA, Kaufman YJ \& Merzlyak MN (1996) Use of a green channel in remote sensing of global vegetation from EOSMODIS. Remote Sensing of Environment, 58:289-298.

Govender M, Govender PJ, Weiersbye IM, Witkowski ETF \& Ahmed F (2009) Review of commonly used remote sensing and ground-based technologies to measure plant water stress. Water SA, 35:741-752.

Haboudane D, Miller JR, Pattey E, Zarco-Tejada PJ \& Strachan IB (2004) Hyperspectral vegetation indices and novel algorithms for predicting green LAI of crop canopies: Modelling and validation in the context of precision agriculture. Remote Sensing of Environment, 90:337-352.

Hillnhütter C, Mahlein AK, Sikora RA \& Oerke EC (2011) Remote sensing to detect plant stress induced by Heterodera schachtii and Rhizoctonia solani in sugar beet fields. Field Crops Research, 122:70-77.

Huete ARA (1988) Soil adjusted vegetation index (SAVI). Remote Sensing of Environment, 25:295-309.

Li G, Wan S, Zhou J, Yang Z \& Qin P (2010) Leaf chlorophyll fluorescence, hyperspectral reflectance, pigments content, malondialdehyde and proline accumulation responses of castor bean (Ricinus communis L.) seedlings to salt stress levels. Industrial crops and products, 31:13-19.

Mahajan GR, Pandey RN, Sahoo RN, Gupta VK, Datta SC \& Kumar D (2016) Monitoring nitrogen, phosphorus and sulphur in hybrid rice (Oryza sativa L.) using hyperspectral remote sensing. Precision Agriculture, 18:736-761.

Mahajan GR, Sahoo RN, Pandey RN, Gupta VK \& Kumar D (2014) Using hyperspectral remote sensing techniques to monitor nitrogen, phosphorus, sulphur and potassium in wheat (Triticum aestivum L.). Precision agriculture, 15:499-522.

Malavolta E, Vitti GC \& Oliveira AS (1997) Avaliação do estado nutricional das plantas: princípios e aplicações. Potafós, Piracicaba. 319p.

Martins GD \& Galo MDLBT (2014) Detection of Infested Areas by Nematodes and Migdolus Fryanus in Sugarcane from Rapideye Multispectral Images. Revista Brasileira de Cartografia, 01:285-301

Melo VF, Ribeiro NA, Maschio PA, Corrêa GF \& Lima VC (2004) Mineralogy and forms of $\mathrm{K}$ and $\mathrm{Mg}$ in different weight and size classes of sand fraction of Triângulo Mineiro soils, Minas Gerais State, Brazil. Revista Brasileira de Ciência do Solo, 28:219231.

Mirik M, Ansley RJ, Price JA, Workneh F \& Rush CM (2013) Remote monitoring of wheat streak mosaic progression using sub-pixel classification of Landsat $5 \mathrm{TM}$ imagery for site specific disease management in winter wheat. Advances in Remote Sensing, 02:16-28.

Mirik M, Jones DC, Price JÁ, Workneh F, Ansley RJ \& Rush CM (2011) Satellite remote sensing of wheat infected by wheat streak mosaic virus. Plant Disease, 95:04-12.

Rev. Ceres, Viçosa, v. 66, n.2, p. 142-153, mar/abr, 2019 
Moreira A \& Fageria NK (2010) Liming influence on soil chemical properties, nutritional status and yield of alfalfa grown in acid soil. Revista Brasileira de Ciência do Solo, 34:1231-1239.

Moreira MA, Adami M \& Rudorff BFT (2004) Spectral and temporal behavior analysis of coffee crop in Landsat images. Pesquisa Agropecuária Brasileira, 39:223-231.

Motomiya AVA, Molin JP, Motomiya WR \& Baio FHR (2012) Mapeamento do índice de vegetação da diferença normalizada em lavoura de algodão. Pesquisa Agropecuária Tropical, 42:112118

Nestel D, Dickschen F \& Altieri MA (1994) Seasonal and spatial population loads of a tropical insect: the case of the coffee leaf-miner in Mexico. Ecological Entomology, 19:159-167.

Ortiz BV, Thomson SJ, Huang Y, Reddy KN \& Ding W (2011) Determination of differences in crop injury from aerial application of glyphosate using vegetation indices. Computers and electronics in agriculture, 77:204-213.

Paiva BRTL, De Souza PE, Scalco MS \& Monteiro FP (2013) Progress of cercospora leaf spot in coffee under different irrigation management systems and planting densities. Coffee Science, 08:166-175.

Peñuelas J, Baret F \& Filella I (1995) Semi-empirical indices to assess carotenoids/chlorophyll a ratio from leaf spectral reflectance. Photosynthetica, 31:221-230.

Pimstein A, Karnieli A, Bansal SK \& Bonfil DJ (2011) Exploring remotely sensed technologies for monitoring wheat potassium and phosphorus using field spectroscopy. Field Crops Research, 121:125-135.

Pozza EA, Carvalho VL \& Chalfoun SM (2010) Sintomas de injúrias causadas por doenças em cafeeiro. In: Guimaraes RJ, Mendes ANG \& Baliza DP (Ed.) Semiologia do cafeeiro: sintomas de desordens nutricionais, fitossanitárias e fisiológicas semiologia do cafeeiro. Lavras, UFLA. p.68-106.

Prabhakar M, Prasad YG \& Rao MN (2012) Remote Sensing of Biotic Stress in Crop Plants and Its Applications for Pest Management. In: Venkateswarlu B, Shanker A, Shanker C \& Maheswari M (Ed.) Crop Stress and its Management: Perspectives and Strategies. Dordrecht, Springer. p.517-545.

Qi J, Chehbouni A, Huete AR, Kerr YH \& Sorooshian SA (1994) Modified soil ajusted vegetation index. Remote Sensing of Environmental, 48:119-126.
Rondeaux G, Steven M \& Baret F (1996) Optimization of soiladjusted vegetation indices. Remote sensing of environment, 55:95-107.

Roujean JL \& Breon FM (1995) Estimating PAR absorbed by vegetation from bidirectional reflectance measurements. Remote Sensing of Environment, 51:375-384.

Rouse JW, Haas RH, Schell JÁ, Deering DW \& Harlan JC (1974) Monitoring the vernal advancement and retrogradation (green wave eff ect) of natural vegetation. Greenbelt, NASA/GSFC. $371 \mathrm{p}$.

Santos FS, Souza PE \& Pozza EA (2004) Epidemiologia da cercosporiose em cafeeiro (Coffea arabica L.) fertirrigado. Summa Phytopathologica, 30:31-37.

Santos WJR, Silva BM, Oliveira GC, Volpato MML, Lima JM, Curi N \& Marques JJ (2014) Soil moisture in the root zone and its relation to plant vigor assessed by remote sensing at management scale. Geoderma, 221:91-95.

Serrano J, Silva JM, Shahidian S, Silva LL, Souza A \& Baptista (2017) Differential vineyard fertilizer management based on nutrient's spatio-temporal variability. Journal of soil science and plant nutrition, 17:46-61.

Silva VMD, Teixeira AFR, Reis EFD, Benassi AC \& Mendonça EDS (2013) Chemical attributes of soil organic fertilizer on systems of coffee conilon. Coffee Science, 08:69-477.

Tola E, Al-gaadi KA, Madugundu R, Zeyada AM, Kayad AG \& Biradar CM (2017) Characterization of spatial variability of soil physicochemical properties and its impact on Rhodes grass productivity. Saudi journal of biological sciences, 24:421-429.

Vasco GB, Pozza EA, Scalco MS, Santos LSD, Custódio AAP \& Silva MLO (2015) Brown eye spot incidence in fruits of coffee: different density planting and water managements. Coffee Science, 10:38-45.

Wójtowicz M, Wójtowicz A \& Piekarczyk J (2016) Application of remote sensing methods in agriculture. Communications in Biometry and Crop Science, 11:31-50.

Zanzarini FV, Pissarra TC, Brandão FJ \& Teixeira DD (2013) Spatial correlation of the vegetation index (NDVI) of a Landsat/ ETM+ images with soil attributes. Revista Brasileira de Engenharia Agrícola e Ambiental, 17:608-614.

Zhao D, Reddy, KR, Kakani VG, Read JJ \& Carter GA (2003) Corn (Zea mays L.) growth, leaf pigment concentration, photosynthesis and leaf hyperspectral reflectance properties as affected by nitrogen supply. Plant and soil, 257:205-218. 